\title{
Consensus Recommendations for Gastric Emptying Scintigraphy: A Joint Report of the American Neurogastroenterology and Motility Society and the Society of Nuclear Medicine
}

Thomas L. Abell, M.D., ${ }^{1}$ Michael Camilleri, M.D., ${ }^{2}$ Kevin Donohoe, M.D., ${ }^{3}$ William L. Hasler, M.D., ${ }^{4}$ Henry C. Lin, M.D., ${ }^{5}$ Alan H. Maurer, M.D., ${ }^{6}$ Richard W. McCallum, M.D., ${ }^{7}$ Thomas Nowak, M.D., ${ }^{8}$ Martin L. Nusynowitz, M.D., ${ }^{9}$ Henry P. Parkman, M.D., ${ }^{10}$ Paul Shreve, M.D., ${ }^{11}$ Lawrence A. Szarka, M.D., ${ }^{2}$ William J. Snape Jr., M.D., ${ }^{12}$ and Harvey A. Ziessman, M.D. ${ }^{13}$

${ }^{1}$ Gastroenterology, University of Mississippi Medical Center, Jackson, Mississippi; ${ }^{2}$ Gastroenterology, Mayo Clinic School of Medicine, Rochester, Minnesota; ${ }^{3}$ Nuclear Medicine, Beth Israel Deaconess Medical Center, Boston, Massachusetts; ${ }^{4}$ Gastroenterology, University of Michigan Medical Center, Ann Arbor, Michigan; ${ }^{5}$ Gastroenterology, University of Southern California, Los Angeles, California; ${ }^{6}$ Nuclear Medicine, Temple University School of Medicine, Philadelphia, Pennsylvania; ${ }^{7}$ Gastroenterology, University of Kansas Medical Center, Kansas City, Kansas; ${ }^{8}$ Central Indiana Gastroenterology Group and Saint Vincent Hospital, Indianapolis, Indiana; ${ }^{9}$ Nuclear Medicine, University of Texas Medical Branch; Galveston, Texas; ${ }^{10}$ Gastroenterology, Temple University School of Medicine, Philadelphia, Pennsylvania; ${ }^{11}$ Advanced Radiology, P.C. and Spectrum Health, Grand Rapids, Michigan; ${ }^{12}$ Gastroenterology, California Pacific Medical Center, San Francisco, California; and ${ }^{13}$ Nuclear Medicine, Johns Hopkins University School of Medicine, Baltimore, Maryland

This consensus statement from the members of the American Neurogastroenterology and Motility Society and the Society of Nuclear Medicine recommends a standardized method for measuring gastric emptying (GE) by scintigraphy. A low-fat, eggwhite meal with imaging at $0,1,2$, and $4 \mathrm{~h}$ after meal ingestion, as described by a published multicenter protocol, provides standardized information about normal and delayed GE. Adoption of this standardized protocol will resolve the lack of uniformity of testing, add reliability and credibility to the results, and improve the clinical utility of the GE test.

J Nucl Med Technol 2008; 36:44-54

DOI: $10.2967 /$ jnmt.107.048116

\section{INTRODUCTION}

Gastric emptying scintigraphy (GES) is commonly performed to evaluate patients with symptoms that suggest an alteration of gastric emptying (GE) and/or motility (1). The first use of radionuclides to measure GE was published in 1966 (2). Since then, it has become the standard for the measurement of gastric motility in clinical practice, because it provides a physiologic, noninvasive, and quantitative measurement of GE (3). After radiolabeling the solid or

Received Jul. 16, 2007; revision accepted Oct. 1, 2007.

Reprint requests and correspondence: Henry P. Parkman, M.D., Gastroenterology Section, Parkinson Pavilion, 8th Floor, Temple University School of Medicine, 3401 North Broad Street, Philadelphia, PA 19140.

(C) 2007 by Am. Coll. of Gastroenterology. liquid component of a meal, the gastric counts measured by scintigraphy correlate directly with the volume of the meal remaining without the need for geometric assumptions about the shape of the stomach. Ultrasound is operator-dependant, requires geometric assumptions, and generally measures only liquid emptying. Other indirect tests of GE include breath testing and acetaminophen absorption. Breath testing indirectly measures GE, as GE is the rate-limiting step in the processing and excretion of ${ }^{13} \mathrm{C}$-octanoic acid. The test assumes normal small bowel absorption and pulmonary function. Acetaminophen absorption test measures liquid GE and requires normal small bowel absorption and requires repetitive blood sampling (3).

While GES has been considered the standard for measuring GE, there is a lack of standardization of the test, including differences in meals used, patient positioning, frequency, and duration of imaging. There are differences in the quantitative data reported, e.g., half-time of emptying, rate of emptying (percent per minute), or the percent retention or emptying at different time points during the study. Normal values often have not been established for some of the protocols used, and the performance characteristics of the test with the specified meal may not have been established or published.

Lack of standardization limits the clinical utility of the test, and presents problems for patients and their physicians as the latter try to interpret study results from other institutions. This often leads to repeat testing using a different protocol with which the gastroenterologist has greater familiarity and 
confidence. This practice presents problems for the patient as insurance companies may not reimburse a second test, or may require the patient to undergo the test at a center where the protocol is suboptimal. Discrepant results between two studies can further complicate patient management.

Several professional societies (the Society of Nuclear Medicine [SNM], American Gastroenterological Association [AGA], and American Neurogastroenterology and Motility Society [ANMS]) have developed guidelines for performing GES (4-7). However, there has not been a consensus on a protocol that meets both the clinicians' needs and the services available at most nuclear medicine facilities. For GES to attain its full clinical potential, there is a need for closer collaboration between nuclear medicine physicians and referring gastroenterologists to develop such a consensus protocol.

This manuscript provides consensus guidelines from members of the ANMS and the SNM. Our goal was to propose a single, standardized protocol for performing GES that meets the needs of clinicians, the imaging specialists, and the patients.

The process leading to this joint report involved three meetings. At the first meeting (Philadelphia, PA, April 2006), the participants focused on the needs of the patient and the referring physician, reviewed current methods considered best practice for performing GES, and addressed unresolved areas requiring further research or clarification. At the second meeting at the SNM annual meeting (San Diego, CA, June 2006), there were presentations and discussion on the same topics, which led to the drafting of a manuscript summarizing a consensus protocol. At a third meeting at the ANMS annual meeting (Boston, MA, September 2006), a consensus protocol was discussed and critiqued. This was followed by final discussions and revisions of this manuscript.

This manuscript represents this group's current recommendation on how to perform a solid-meal GES test for clinical practice, using readily available technology and normative data, which can provide clinicians with standardized results. Throughout this process, we have recognized that the current consensus may not imply unanimity of opinion. In addition, this document does not answer all the questions raised during these discussions.

However, this manuscript addresses those aspects that the multidisciplinary group considers were in the greatest need of immediate standardization - the meal, the frequency of imaging, the duration of the test, and the normative data. Technical issues concerning the acquisition and processing parameters are less controversial and do not contribute to wide variations in methodology. These have been addressed elsewhere $(5,6)$, and are summarized in Appendix 1. The manuscript also identifies areas for future study, but there was an overwhelming consensus on the pressing need to recommend a standardized GES test.

\section{DISORDERS OF GE}

Both rapid and delayed GE can cause similar symptoms. Hence, it is important to evaluate patients for both rapid and delayed GE in the same test. It is, therefore, appropriate to review some of the relevant clinical information on the symptoms related to the disorders of GE.

\section{Delayed GE}

Gastroparesis is diagnosed when symptoms such as nausea, vomiting, early satiety, postprandial fullness, abdominal discomfort, and pain are associated with objective evidence of delayed GE in the absence of obstruction, and typically with impairment in maintenance of normal nutrition using standard food $(4,8)$. These symptoms are also, as reported by some individuals, associated with the postprandial distress subtype of functional dyspepsia (9). Symptoms that suggest delayed GE in patients with dyspepsia are primarily postprandial fullness, nausea, and vomiting $(10,11)$. In patients with diabetes, symptoms that have been associated with delayed GE are abdominal bloating/fullness and upper abdominal pain $(12,13)$. Some studies, however, have shown a poor correlation between the rate of solid GE and the severity of gastric symptoms (12-14). Furthermore, some clinical trials of drug therapy for gastroparesis show variable symptomatic benefit from pharmacologic stimulation of GE $(15,16)$. In addition, some patients' symptoms improve with prokinetic agents, but GE remains unchanged (16). Investigations are attempting to determine if factors other than a global delay in GE such as impaired fundic accommodation, antral distension, antral hypomotility, gastric dysrhythmias, visceral hypersensitivity, or psychological disturbances explain, in part, the symptoms experienced by patients with suspected gastroparesis (1).

Many scoring systems have been used for the determination of symptom severity in patients with possible gastroparesis. Symptoms of gastroparesis may be quantified by a validated symptom questionnaire, the gastroparesis cardinal symptom index (GCSI) $(17,18)$. The GCSI is based on three subscales (postprandial fullness/early satiety, nausea/vomiting, and bloating) and represents a subset of the longer patient assessment of upper gastrointestinal disorderssymptoms (PAGI-SYM) that quantifies symptoms of gastroparesis, dyspepsia, and gastroesophageal reflux disease (19).

\section{Rapid GE}

Rapid GE is the major factor in dumping syndrome, characteristically seen after surgery for peptic ulcer disease with and without vagotomy $(20,21)$. The early symptoms of "dumping" occurring in the initial hour after meal ingestion include diarrhea, abdominal discomfort, nausea, bloating, and vasomotor symptoms. Some of these symptoms may be difficult to distinguish from the gastroparesis symptoms. The late dumping syndrome presents with diaphoresis, palpitations, weakness, and fainting, which are secondary to reactive hypoglycemia from exaggerated insulin release.

Rapid GE of solids has been demonstrated in some patients with unexplained nausea, bloating, and fullness (22). Rapid GE has recently been reported in some patients with functional dyspepsia (23). Rapid GE also occurs in some diabetic patients, particularly in the early stages of 
type II diabetes (24). Many of these patients have symptoms indistinguishable from those of gastroparesis. Rapid movement of food from the stomach into the small bowel with small bowel distension may explain pain and nausea, symptoms similar to those described in patients with delayed GE. Rapid emptying has been recently observed as an accompanying factor in adult patients with cyclic vomiting syndrome $(25,26)$. In one study, rapid GE was more common than delayed GE in symptomatic patients with autonomic dysfunction (27).

\section{FACTORS TO CONSIDER IN THE MEASUREMENT OF GE}

Measurements of GE are influenced by a variety of factors, which must be considered when performing GES (28). Factors that may influence the performance and negatively influence the clinical validity of a GES are (a) short duration of testing, (b) extrapolated T-1/2 measurements, (c) unknown meal composition and amount consumed, (d) medications at the time of the test, (e) poor or unknown glycemic control at the time of the test, and (f) vomiting a portion of the meal.

\section{Patient-Related Factors to be Considered for GE Tests}

Patient-related factors that need to be considered in GE tests include medications, tobacco smoking, hyperglycemia, and gender $(1,3)$.

Patients may be taking medications to intentionally affect GE (e.g., prokinetic agents) or mediations that alter emptying as a side effect (e.g., narcotic analgesics). Depending on the reason for the test, patients may need to discontinue these medications. The period of time off medication before the test should be based on the drug's half-life, for most medications, this will be 48-72 h (3). Opiate analgesic medications and anticholinergic agents delay GE and, if not discontinued prior to the study, could result in a false diagnosis of delayed GE. Prokinetics may lead to a normal GE result in a patient with gastroparesis. Serotonin receptor (5-HT-3) antagonists, such as ondansetron, which have little effect on GE, may be given for severe symptoms of nausea and vomiting before performance of GES (29).

Tobacco smoking has been shown in early studies to slow GE $(30,31)$. This effect may not be related to nicotine (31, 32). Recent studies with ${ }^{13} \mathrm{C}$-octanoic breath testing, however, have shown conflicting results (33). It is recommended that patients abstain from smoking in the morning of the test and throughout the time GE is being imaged.

Hyperglycemia can delay GE. Although some data suggest that even modest degrees of hyperglycemia ( $\geq 144 \mathrm{mg} / \mathrm{dL}$ ) retard GE (34), it is not clear what level produces clinically significant delay in GE (35). Marked hyperglycemia with serum glucose levels $\geq 288 \mathrm{mg} / \mathrm{dL}$ significantly delays GE in diabetic patients when compared with euglycemia (36). The general consensus is that blood glucose should be under reasonable control on the day of a GES to obtain a reliable measurement of GE. For diabetics, it is recommended that serum glucose be measured prior to the study, noted in the report, and if the blood glucose is $>275 \mathrm{mg} / \mathrm{dL}$ on the morning of the test, the glucose should be lowered with insulin to $<275 \mathrm{mg} / \mathrm{dL}$, or the study should be rescheduled for another day when the blood glucose is under better control. Some centers administer insulin if the blood glucose level immediately before GES is $>180 \mathrm{mg} / \mathrm{dL}$ and do not start the test until the glucose is $<180 \mathrm{mg} / \mathrm{dL}$ (Horowitz, personal communication).

Premenopausal women are reported in some studies to have slower GE than men $(37,38)$. A few centers use separate reference values for premenopausal women (10), or limit testing to the first week of the menstrual cycle before estrogen and progesterone peak. The latter approach is suggested, as separate values are not generally available for men and women. The 4-h test of Tougas et al., recommended in these guidelines, did not show variability attributable to menstrual phase and gender (39). A study of postmenopausal women administered estrogen or progesterone or a combination to mimic normal hormone levels in premenopausal women did not demonstrate any effect of these sex hormones on GE (40).

\section{Technical Factors in GE Tests}

There are many reasons for the current diversity of imaging and analysis of GES protocols: an individual center's meal and analysis preferences, different camera and computer systems, scheduling constraints, and processing software.

Solid-phase GES is used to document gastroparesis. Liquid GE tests are generally not clinically useful, because normal emptying of liquids is frequently maintained despite very severe gastroparesis for solids (41). Meals currently used for measuring GE consist of a variety of foods, including chicken liver, eggs, egg whites, oatmeal, or pancakes. The content of the meal used is one of the most important variables needing standardization because GE depends on meal composition. Solids empty more slowly than liquids with digestible solids emptying more rapidly than indigestible residue (1). Additionally, emptying of fats is slow as compared to emptying of proteins or carbohydrates. The caloric content and volume of the test meal will also alter GE. Incomplete meal ingestion can lead to values suggesting more rapid emptying. Vomiting a portion of the ingested meal after the initial baseline image may lead to lower subsequent estimated gastric retention values, so that GE appears faster than it was.

Reported values of GE are influenced by the duration of testing and the method of analysis. Half-times (T-1/2 values) of emptying may be potentially less accurate than percentages of retention measured at fixed time points, particularly in individuals with very prolonged emptying in which extrapolation is needed to calculate the half-time if it was not actually reached during the test. Surveys of nuclear medicine centers show wide variations in reporting rates of emptying. In a study of Canadian health-care facilities, $40 \%$ of the centers did not validate a normal range for their 
GE test meal and the range of normal values varied considerably: $20 \%$ of the centers used two standard deviations (SD) about the mean, 26\% used one SD, and 6\% used 1.5 SD for normal ranges (42).

Individual preferences of both referring physicians and imaging specialists have influenced local decisions on how to conduct GES. Currently, studies vary in length from as short as $60 \mathrm{~min}$ up to $4 \mathrm{~h}$. Studies have shown that extending measurements out to $4 \mathrm{~h}$ increases the detection rate of delayed emptying $(43,44)$. As more has been learned about the optimal methodology, some imaging centers have changed their protocols, but many centers have been reluctant to perform a 4-h procedure because reimbursement is not commensurate with the time and effort needed. Reimbursement for GES is substantially lower than that for other imaging procedures that can be done in less time on the same equipment. This economic reality, however, should not be an impediment in providing optimum patient care. Under current Center for Medicare and Medicaid Services (CMS) guidelines, any future increase in relative value unit (RVU) assignment (reimbursement) will not occur until the medical community demonstrates a consensus on the requirement for more comprehensive imaging.

\section{TOWARD STANDARDIZATION: VALIDATION OF THE PROPOSED REGIMEN}

One multi-institutional protocol has been published which investigated a large number of normal subjects and established normal values (39). One hundred twenty-three normal subjects from 11 medical institutions in the United States, Canada, and Europe were studied in a multi-institutional and multinational study. Normal values published from other protocols were often based on smaller numbers of subjects (typically 10-35) at single institutions (45). The percent GE was quantified at several time intervals after consumption of a low-fat, egg-white meal. The Technetium (Tc)-99m sulfur colloid radiolabeled meal consisted of the equivalent of two large eggs (Eggbeaters ${ }^{\circledR}$, ConAgra Foods, Inc., Omaha, NE), two slices of bread and jam with water. Imaging was performed in the anterior and posterior projections at only four time points $(0,1,2$, and $4 \mathrm{~h})$. The geometric mean activity of decay-corrected counts (square root of the product of the anterior and posterior counts) was determined at each imaging time. Normal values were established using the median and 95th percentile because the data were skewed, particularly at $4 \mathrm{~h}$. These authors defined the upper limits of gastric retention (95th percentile) of the 123 men and women at each of three time points: 1,2 , and $4 \mathrm{~h}$ (Table 1 ). Delayed GE (gastric retention) was determined to be $>90 \%$ at $1 \mathrm{~h}$, $>60 \%$ at $2 \mathrm{~h}$, and $>10 \%$ gastric retention at $4 \mathrm{~h}$. These results currently provide the largest published database for a standardized GES protocol (39).

Imaging at only four time points can actually simplify scheduling and permit more efficient use of imaging equipment. This helps alleviate some of the concerns at some imaging centers that GES is too time-consuming, and
TABLE 1

Normal Values for Low-Fat, Egg-White Gastric Emptying Scintigraphy

\begin{tabular}{|c|c|c|}
\hline $\begin{array}{l}\text { Time } \\
\text { Point }\end{array}$ & $\begin{array}{l}\text { Lower Normal Limit for } \\
\text { Gastric Retention }\end{array}$ & $\begin{array}{l}\text { Upper Normal Limit for } \\
\text { Gastric Retention }\end{array}$ \\
\hline $0 \mathrm{~min}$ & $\begin{array}{l}\text { A lower value suggests } \\
\text { rapid gastric emptying }\end{array}$ & $\begin{array}{l}\text { A greater value suggests } \\
\text { delayed gastric emptying }\end{array}$ \\
\hline $0.5 \mathrm{~h}$ & $70 \%{ }^{\dagger}$ & \\
\hline $1 \mathrm{~h}$ & $30 \%{ }^{\dagger}$ & $90 \% *$ \\
\hline $2 \mathrm{~h}$ & & $60 \%$ * \\
\hline $3 \mathrm{~h}$ & & $30 \%{ }^{\ddagger}$ \\
\hline $4 \mathrm{~h}$ & & $10 \%$ * \\
\hline \multicolumn{3}{|c|}{$\begin{array}{l}\text { Values are the 95th percentile confidence interval. } \\
{ }^{\star} \text { Tougas et al. (39). } \\
{ }^{\dagger} \text { Abell et al. (50). } \\
{ }^{\ddagger} \text { Lin et al. (51). }\end{array}$} \\
\hline
\end{tabular}

the efficiencies in camera use are associated with little loss of test accuracy $(46,47)$. Multiple GE tests can be performed on one camera in a single day if the starting times are staggered.

Another important factor supporting use of Touga et al.'s protocol is the data suggesting the superiority of a longer, 4-h study rather than a 2 -h study $(39,43,44)$. The first reported studies promoting 4-h imaging were from the Mayo Clinic in 1991 and $1995(47,48)$. In a study of 35 patients, the 4-h time interval was more sensitive for detection of delayed GE than the 2-h time point. The Temple University group reported in 2001 on 127 consecutive patients referred for clinical GE studies (43). GES was performed with a conventional egg labeled sandwich with water. Imaging was performed at 0 , $0.5,1,2,3$, and $4 \mathrm{~h}$. The data suggested that the 3 - and 4-h imaging times detected more abnormal GE than 2-h images, e.g., $50 \%$ of the patients were abnormal at $3-4 \mathrm{~h}$ versus $33 \%$ at $2 \mathrm{~h}$. Data from Johns Hopkins University have found similar results using the Tougas et al. meal $(44,49)$. In an investigation of 175 patients, 34 studies were abnormal at $2 \mathrm{~h}$. The 4-h retention identified 11 additional abnormal patients who had normal emptying at $2 \mathrm{~h}$, a $29 \%$ increase in the number of abnormal studies.

Any GES protocol should be able to identify both rapid and slow GE. Although the symptoms of rapid emptying may be similar to those of delayed emptying (23), the treatment is quite different. Use of a standardized protocol across all medical centers that includes standard time points (e.g., 0, 1, 2 , and $4 \mathrm{~h}$ ) offers the potential of consistently defining both delayed and rapid GE. The early 1-h time point can be used for determining rapid GE; whereas the later 2- and 4-h time points are used for determining delayed GE. The study of Tougas et al. did not establish values for rapid emptying. Reanalysis of data from the original Tougas et al. manuscript suggests $32 \%$ is the lower $95 \%$ confidence interval (CI) for normal emptying, suggesting that $<30 \%$ retention at $1 \mathrm{~h} \mathrm{(50)}$ is indicative of rapid GE (Table 1). 


\section{ITEMS FOR FURTHER INVESTIGATION}

It is anticipated that GES will need to be further optimized as more studies using this protocol become available. The consensus participants, therefore, identified the following areas where more information is needed. A list of these items is summarized in Table 2.

\section{Optimal Timing of Imaging}

Some patients with delayed emptying at $2 \mathrm{~h}$ normalize their emptying at the 4-h time point and some individuals with normal emptying at $2 \mathrm{~h}$ have delayed emptying at $4 \mathrm{~h}$ $(43,44)$. The clinical importance of delayed emptying at only certain time points is unknown. There needs to be a better understanding of the use of multiple time points in combination (e.g., 2 and $4 \mathrm{~h}$ ). Data from Guo et al. suggest that the

TABLE 2

Issues Requiring Further Investigation for GES

1. Optimization of the specific time points used for imaging and interpretation:

A. Use of 0.5 - or 1-h result for detection of rapid gastric emptying.

B. Use of 3-h result compared to 2- and 4-h results for detection of delayed GE.

C. Use of multiple time points (2- and 4-h) versus single 2- or 4-h values and further understanding of the clinical meaning of discordant results between 2- and 4-h scans.

2. Need for normal data on other meals:

A. Use of different composition solid meals with different caloric/fat challenges.

B. Need for alternative meals for patients unable to tolerate eggs, allergic to eggs, or with gluten sensitive enteropathy.

3. Need for glycemic control and management of diabetic patients:

A. Assessment of glucose in diabetic patients prior to the test: glucose and Hgb-A1c.

B. Management of hyperglycemic patients on the day of test.

C. Administration of insulin and oral hypoglycemic agents.

D. Need for monitoring postprandial glucose.

4. Value of monitoring symptoms during the time of study.

5. Development of a scale to assess severity of delayed gastric emptying.

6. Need for database of "normal" values for postgastric surgery patients.

7. Clinical value of characterization of proximal and distal gastric function:

A. Regional analysis of gastric emptying (separate antral and fundal measurements).

B. Dynamic antral contraction studies.

C. Fundal accommodation studies with SPECT.

8. Other quantitative measurements:

A. Curve fitting.

B. Lag phase measurements.

C. Use of total abdominal counts.

9. Industry software development:

A. Need for industry to develop commercial acquisition and processing protocols that support these consensus recommendations. 3-h time period might be as sensitive as a 4-h study in detecting delayed GE (43). Although the original report for the Tougas et al. data had no 3-h measurement point, more recent studies suggest that the upper limit of normal is $28 \%$ gastric retention at $3 \mathrm{~h}$ after meal ingestion (51). Another study suggests that $30 \%$ is the optimal threshold for 3-h emptying data (44). A recent study using the Tougas et al. meal has shown that the 3-h time point is nearly comparable to the 4-h value in detecting patients with delayed GE (51).

For rapid GE, currently, the 1-h GE value is recommended. However, the normal values were generated with a smaller subgroup of normal subjects, and are currently published only in abstract form for the Tougas et al. meal (50). More data are needed for delineation of the cutoffs to determine rapid GE. Recent investigations also suggest that rapid emptying is detected at $15-60 \mathrm{~min}$ after meal ingestion (22, 23). It is conceivable that earlier time points, such as a 30-min postprandial value, may be better than the 1 -h value for detection of rapid GE.

The time points for measuring GE may be further optimized in the future. Presently, however, there are insufficient data to recommend the 30-min and the 3-h time points for routine use (Table 2). The current consensus recommendation is to obtain images, at a minimum, at $0,1,2$, and 4 hours after meal ingestion as described originally (39). More images can be obtained, if desired.

\section{Composition of Meal}

This consensus document proposes a low-fat meal used as the initial screening test for GE. In some patients, a low-fat, egg-white meal may not prove to be an adequate functional challenge, especially for patients who report symptom exacerbations after eating lipid-rich foods (22). Meal composition may need to be altered depending on the patient's specific symptoms. Some European investigators have suggested adding butter $(10 \mathrm{~g})$ to the low-fat meal to increase the fat content. This brings the meal to a caloric content of $345 \mathrm{kcal}$ with nutritional composition of $69 \%$ carbohydrate, $22 \%$ protein, $7 \%$ fat, and $2 \%$ fiber. Other alternative meals may also be useful for patients with egg allergies or intolerance to eggs, and patients with gluten-sensitive enteropathy. Liquid Ensure ${ }^{\circledR}$ (Abbott Laboratories, Abbott Park, IL) nutrient supplement or an oatmeal meal is used by some centers. However, specific normal databases will be needed for these alternative meals before they are used clinically.

\section{Glycemic Control}

More uniform management of diabetic patients undergoing GES is needed. The consensus opinion is to measure and record the blood glucose level of diabetic patients just prior to the study. The study should be performed if the patient is under reasonable glucose control, that is, fasting glucose of $<275 \mathrm{mg} / \mathrm{dL}$. There is still no consensus, however, on what should be done with insulin and oral hypoglycemic agents on the day of the study. Generally, half of 
the usual morning dose of insulin is given with the radiolabeled test meal. Postprandial glucose is not usually measured to determine if hyperglycemia develops postprandially during the GE test; postprandial hyperglycemia could prolong GE. Hypoglycemia may also influence GE or increase the patient's symptoms. Postprandial glucose monitoring at 2 and $4 \mathrm{~h}$ may be considered in the future.

\section{Monitoring of Symptoms}

Monitoring of symptoms during the GE test is currently being performed in clinical research studies in both diabetic and nondiabetic patients $(13,52)$ to determine whether alterations in GE correlate with symptoms. Clinically, if the GE test is normal, this simultaneous recording of symptoms will inform whether the meal was an appropriate provocative test to induce the typical postprandial symptoms.

\section{Assessment of Severity}

Because there is not a close correlation between the delay in GE and the symptoms, the GE test alone should probably not be used for grading the severity of the clinical disorder of gastroparesis. Grading the severity of the delay in GE has been performed in clinical research studies and might be used clinically (52). Grading for severity of delayed GE based on the 4-h value in groups related to the SD of the normal results is: grade 1 (mild): $11-20 \%$ retention at $4 \mathrm{~h}$; grade 2 (moderate): $21-35 \%$ retention at $4 \mathrm{~h}$; grade 3 (severe): $36-50 \%$ retention at $4 \mathrm{~h}$; and grade 4 (very severe): $>50 \%$ retention at $4 \mathrm{~h}$.

On the other hand, a combination of the degree of GE delay and the nutritional needs or approaches necessary to support the patient's hydration and nutrition provide a better assessment of severity and facilitate the approach to management. In a recent review, mild delay was designated as $11-15 \%$, moderate $16-35 \%$, and severe $>35 \%$ retention at $4 \mathrm{~h}(53)$.

\section{Establishing Normal Values for the Postgastric Surgery Group}

There is no appropriate "normal" database to use for patients that are symptomatic after gastric surgical procedures. In postsurgical patients, the altered anatomy is likely to alter GE as compared to normal subjects without gastric surgery (54). In general, there is a delay in the emptying of solids and an accelerated emptying of liquids (54). After Roux gastrectomy, there may be retention of solids in both the gastric remnant and the Roux limb (55). The normal GE of the Tougas et al. meal in patients with different degrees of partial gastric resections (e.g., antrectomy) and different drainage procedures is not known. This is also relevant with surgical reconstruction of the stomach, especially postbariatric surgery of the stomach.

\section{Assessment of Proximal and Distal Gastric Function}

A unique advantage of GES is that it can characterize more completely the complex physiology of GE including proximal (fundal) and distal (antral) function. Scintigraphy permits analysis of the intragastric distribution of the test meal between the fundus and the antrum. Routine GE imaging can be used to measure both regional and total GE. Visual inspection of fundal and antral GE and quantification of regional emptying with fundic and antral regions of interest can be helpful for defining abnormal physiology and explaining dyspeptic symptoms, especially when global GE values are normal (56-59). Studies have shown an association between symptoms of nausea, early satiety, abdominal distention, and acid reflux and proximal gastric retention; whereas vomiting is associated more with delayed distal GE (57). However, more validation of intragastric distribution and regional emptying is necessary before this is incorporated into clinical practice.

\section{Other Quantitative Measurements (Lag Phase, Curve Fitting, and Total Abdominal Counts)}

The lag phase is the time required for the commencement of GE of solid particles. It represents the time for the solid food to be triturated into small particles that are then passed through the pylorus. The lag phase can be measured as the time from meal ingestion to the first appearance of the radiolabeled solids in the proximal small bowel. This approach often requires frequent imaging for at least the first $60 \mathrm{~min}$ of the GE study, as normal values have been reported to be $20 \pm 10 \mathrm{~min}$ (SD) (60). It is not certain whether this physiological information is important for clinical management. Gastroparesis is often associated with a prolonged lag phase and slow postlag emptying rate (61). However, a prolonged solid GE lag phase has been implicated in some investigations as the cause of delayed emptying in certain diseases and shortening of the lag phase by prokinetic drugs as the reason for improved emptying (61). One report has suggested the lag phase to be a sensitive indicator of the efficacy of drugs used to treat diabetic gastroparesis (62). Recent reports with the Tougas et al. meal suggest that the length of the lag phase does not correlate with GE and does not add additional information to the percent retention at specified times (44).

Curve fitting to the data points for gastric retention over time may provide useful information on GE (63-66). The GE curve can be analyzed in several mathematical ways to determine both the emptying rate and the lag phase. A curve fitting procedure such as a dual exponential equation (modified power exponential) has been used, namely, $y=100[1-$ $\left.\left(1-e^{-k t}\right)^{b}\right]$ where $y$ is the percent remaining at time $t, k$ is an exponential emptying rate constant (fraction of amount present at time $t$ ), and $b$ is the $y$-intercept of the terminal exponential with slope $=-k$. The lag time can be then calculated as $\ln (b) / k$. Slope and lag time may be helpful to interpret borderline results. Using curve fitting techniques and a similar meal to the low-fat Tougas et al. meal, the upper limit of normal for the lag phase is approximately $45 \mathrm{~min}$ (65). If considering the curve fitting approach for gastric interpretation, several additional data points should be obtained such that imaging occurs at $0,0.5,1,2,3$, and $4 \mathrm{~h}$.

Recently, several studies have suggested that measurement of GE calculated using the proportion of gastric counts as compared to the total abdominal counts might be 
an alternative type of analysis to the conventional method of using only intragastric counts. To date, however, there are only limited data published on this approach $(67,68)$.

\section{Need for Commercial GE Software}

Currently, there is also no uniformity in the commercial software available for processing GE studies. The consensus group anticipates that with the adoption of this consensus protocol, the industry that produces nuclear medicine cameras and computers will need to develop software for acquisition and processing of studies to meet this new standard.

\section{SUMMARY CONSENSUS RECOMMENDATIONS FOR A STANDARDIZED GE PROCEDURE}

This consensus document concludes that, at the current time, the most universally acceptable meal is the low-fat, egg-white meal as described by Tougas et al. Eggbeaters ${ }^{\circledR}$ or the other equivalent generic commercial brands of liquid egg white are available and acceptable for use. Currently, this meal and the protocol have the largest normative database (39). GES should be performed with imaging, at a minimum, at $0,1,2$, and $4 \mathrm{~h}$ after radiolabeled meal ingestion.

Ultimately, to have value, any standardized protocol must be followed closely. Our current consensus recommendation, including the technical details on how to perform GES, is presented in Appendix 1. In order to facilitate better standardization, a number of sample documents are presented. A patient information form for those undergoing the test is contained in Appendix 2 (Reference: ANMS Web site: http://www.motilitysociety.org). A form for all patients to complete to provide important information to assist in study interpretation is contained in Appendix 3.

This consensus statement recommends use of a single, standardized GES protocol. Adoption of this standard is important to improve how GE studies are used to direct patient care. The authors recognize that any consensus protocol has limitations. However, the one currently recommended has the largest database of normal values. Adopting this protocol will solve the problem of nonuniformity of protocols across institutions and will be a vast improvement over the diverse methods currently in use. Other questions and issues will need to be addressed in the months and years ahead, before GES attains its full clinical potential (Table 2). With continued close collaboration between imaging specialists and gastroenterologists, this important physiologic test will continue to serve as a valuable tool in the diagnosis and management of gastric motility disorders.

\section{APPENDIX 1: CONSENSUS METHOD FOR PERFORMING GASTRIC EMPTYING SCINTIGRAPHY}

\section{Patient Preparation}

The referring physician should determine what medications are to be continued prior to GES.

Prokinetic agents that enhance gastric emptying (GE) such as metoclopramide (Reglan), tegaserod (Zelnorm), erythromycin, and domperidone (Motilium) are generally stopped at least 2 days prior to the test. Opiate analgesic medications delay GE and should also be stopped 2 days before the test. These include: Demerol, codeine, morphine, Oxycontin, Percodan, and Percocet.

Anticholinergic antispasmodic agents such as Bentyl, Donnatal, Levsin, and Robinul are usually stopped for 2 days prior to the test.

It is preferable to study menstruating females during the first 10 days of their menstrual cycle to improve the interpretation of the study and to prevent administration of radionuclide to a potentially pregnant woman.

The study should be performed in the morning after an overnight fast. If this is not possible, patients should be fasting for at least $6 \mathrm{~h}$ prior to the test. The patient may take medications with some water on arising before coming for the test. Patients should refrain from smoking in the morning of the test and throughout the time of imaging.

Diabetic patients should test and record their blood glucose prior to the study (fasting). Generally, the fasting glucose should be $<275 \mathrm{mg} / \mathrm{dL}$. At the time the meal is ingested, diabetic patients should self-administer their insulin at a dose that is generally half of what they normally take.

Subjects should be instructed that they will be in the imaging facility for at least $4 \mathrm{~h}$ after meal ingestion and are advised to bring reading material, a personal music player, or other materials to occupy them for the time of the study. For the time between images, the subjects can be sitting, standing, or walking but should remain in close proximity to the nuclear medicine section.

\section{Meal Preparation and Ingestion}

The standard scintigraphic meal for GE should consist of an egg-white meal (Egg Beaters ${ }^{\circledR}$ or generic equivalent) radiolabeled with $0.5-1 \mathrm{mCi}{ }^{99 \mathrm{~m}} \mathrm{Tc}$. The meal has a caloric value of $255 \mathrm{kcal}$ (nutritional composition: $72 \%$ carbohydrate, $24 \%$ protein, $2 \%$ fat, and $2 \%$ fiber). An allergy to eggs is a contraindication to this meal.

Items needed for GE meal:

$4 \mathrm{oz}$. (120 g, equal to approximately two large eggs) liquid egg white (99\% real eggs, cholesterol-free, fat-free, and low-calorie); two slices of white bread (120 kcal), strawberry jam (30 g, $74 \mathrm{kcal})$, water $(120 \mathrm{~mL})$, and technetium-99m sulfur colloid, $0.5-1 \mathrm{mCi}$.

The eggs can be cooked either scrambled on a hot skillet (nonstick frying pan) or microwaved in an appropriately shielded container. The eggs are served with toast, jam, and water. To prepare the meal, the liquid egg is poured into a bowl, mixed with $0.5-1 \mathrm{mCi}{ }^{99} \mathrm{Tc}$ sulfur-colloid marker, and cooked in a skillet (nonstick frying pan) or microwave. The egg mixture should be stirred once or twice during cooking and is cooked until it has the consistency of an omelet (3-5 min). The bread is toasted. Jelly is spread on the bread, and a sandwich is made of the jellied bread and cooked egg mixture. 
The subject ingests the sandwich meal within $10 \mathrm{~min}$. Patients may eat the egg and toast/jelly separately. For quality control, the staff technologist records how long it takes the subject to consume the meal and how much they consume. The patient should ingest the whole meal. If the patient cannot eat the entire meal, at least $50 \%$ of each component should be consumed for the test. If the patient vomits part of the meal at any time during the test, this should be indicated on the report.

\section{Image Acquisition}

Gamma camera images are acquired using a $140 \mathrm{keV}$ ${ }^{99} \mathrm{Tc}$ photopeak with a $20 \%$ window $(140 \mathrm{keV} \pm 10 \%)$. A low-energy all-purpose (LEAP) collimator will maximize the count rate; a low-energy high-resolution collimator can also be used. Computerized digital images are required for quantification. These are acquired in a $128 \times 128$ word mode matrix.

Gamma camera images are obtained immediately after meal ingestion and at a minimum of 1,2 , and $4 \mathrm{~h}$ after meal ingestion with the subject standing upright in front of a gamma camera. One-minute anterior and 1-min posterior timed images are acquired. These may be simultaneous if a dual-headed camera is available. If a single-headed camera is used, the subject is first imaged anteriorly and then posteriorly. For patients who cannot stand or otherwise be positioned for anterior and posterior views, a single-best, left anterior oblique (LAO) image may be substituted.

The time of the images and time to eat the meal should be recorded by the technologist to make sure imaging at specific times is adhered to.

Between imaging sessions, the subjects are permitted to sit in a designated waiting area and to walk to and from the imaging room and bathroom as desired. Strenuous activity (climbing stairs, other physician visits, or other diagnostic studies) should not be scheduled concurrently.

\section{Image Analysis and Quantification of GE}

Manual regions of interest are drawn on the anterior and posterior images for all acquisition times using an irregular region of interest (ROI) tool to outline the stomach. The total gastric ROI should include the fundus and antrum with particular attention to avoid any loops of small bowel in close proximity to the stomach. An exception would be if the patient has small bowel activity on the first image, then the entire field of view should be used so that time 0 includes all activity ingested.

The geometric mean (GM) of the anterior and posterior gastric counts for each time point is calculated and corrected for ${ }^{99 \mathrm{~m}} \mathrm{Tc}$ decay $(6.02 \mathrm{~h}$ half-life) where the GM count $=$ (anterior counts $\times$ posterior counts $)^{1 / 2}$. No geometric mean attenuation correction is required if the patient cannot stand for anterior and posterior views and a single LAO view only is obtained.

The final results are expressed as percent remaining in the stomach at each time point with the total gastric counts normalized to $100 \%$ for the time $t=0$ (first image set immediately after meal ingestion).

\section{Reporting}

The percent remaining in the stomach at each time point is reported. The report should contain the normal values at the key time points: $1 \mathrm{~h}(37-90 \%), 2 \mathrm{~h}(30-60 \%)$, and $4 \mathrm{~h}$ $(0-10 \%)$. A graph of the values plotted as a function of time may also be included in the report as a visual summary of the study results.

The report should mention the fasting blood glucose if the patient is diabetic. The report should mention medications that the patient was taking within the last $24 \mathrm{~h}$ of the test that may affect GE.

The report should document the amount of the meal ingested, the total time taken to ingest the meal, and if any vomiting of the meal occurred postprandially.

The report should also describe any other unusual findings which are observed in the images such as: abnormal esophageal retention of the meal, hiatal hernia, fundal wrap, lack of fundic accommodation, evidence for retained old food particles.

\section{APPENDIX 2: SAMPLE PATIENT INSTRUCTION SHEET FOR GASTRIC EMPTYING SCINTIGRAPHY}

Your doctor has ordered a test that will permit evaluation of how food moves through your stomach. These studies will be performed in the nuclear medicine department.

Preparation for the GE Test:

- You should not eat any food after midnight, the night before the test. If you smoke, do not smoke, beginning on the morning of your test and throughout the time you are having the pictures of GE recorded. You may smoke after you are instructed that the test is completed.

- Some medications are generally stopped for this test. This should be discussed with your doctor or healthcare provider. Drugs that affect GE such as Reglan (metoclopramide), Zelnorm (tegaserod), erythromycin, Motilium (domperidone), and antispasmodics such as Bentyl, Donnatal, Levsin, and Robinul are usually stopped for 3 days prior to this test. Do not take any laxatives on the day before or any time during your study.

- Unless otherwise directed by your doctor, the following pain medications should not be taken for 2 days prior to your test: Pain medications such as Demerol, codeine, morphine, Oxycontin, Percodan, and Percocet sedatives or tranquilizers, such as Valium, Librax, Ativan, or Thorazine.

- Unless otherwise directed by your physician, you may continue your normal medications that could be taken with a small amount of water or juice up to $2 \mathrm{~h}$ prior to your study. You should not drink coffee or tea. 
- If you have diabetes, skipping breakfast may affect your need for diabetic medication. If you are a diabetic and on insulin, we request that you bring your regular morning dose of insulin with you. You can take this with the meal that will be given to you. We may reduce your insulin dose to adjust for the small size of the breakfast. Often half of your insulin is taken with the test meal. If you take oral hypoglycemic medications, generally these are taken with the meal in the nuclear medicine department. If there are any questions concerning your dose of insulin this should be discussed with your physician, radiologist, or the nuclear medicine department's physician performing the test.

- If you have diabetes, we also ask you to bring your glucose monitoring equipment to the test. We will ask you to check your glucose before the test and possibly during or after the test.

- Women, please note: This test should not be performed if you are pregnant. Inform your physician or nurse if you are pregnant or think you may be pregnant. You will be asked if there is the possibility of pregnancy. Often the test is best scheduled for females during the first 10 days of the menstrual cycle.

Description of the GE Test:

- For this test, you will be asked to eat an egg meal that consists of the equivalent of two eggs on toast together with water and jelly. The meal has been labeled with an isotope that will permit pictures to be taken as the meal passes through the stomach and the GI tract.

- Pictures of short duration are acquired with you standing in front of the nuclear medicine department's gamma camera. Between the images you will be permitted to walk about and continue normal activities. It is suggested that you bring some reading material and/or a "Walkman" or an "iPod" if you have personal music preferences. These studies try to simulate normal daily activities. The nuclear medicine department's rooms may be cooler than the rest of the hospital, and you may want to bring a sweater with you.

- The GE test generally takes $4 \mathrm{~h}$ once it is started.

\section{APPENDIX 3: SAMPLE NUCLEAR MEDICINE INFOR- MATION SHEET FOR PATIENTS UNDERGOING GAS- TRIC EMPTYING SCINTIGRAPHY TO FILL OUT}

Your name: Today's date: Your doctor:

Your age: Your weight: Your height:

1. What is your main symptom for undergoing the GE test today?

(Please circle the best answer or write in):

Heartburn Chest pain Nausea Vomiting

Abdominal pain Bloating/Distension Constipation Diarrhea

Other symptom:
2. Do you have diabetes? No Yes

If yes, how long have you had diabetes? years

What medications do you take: insulin pills

Did you measure your glucose this morning before the test? No Yes

If yes, what was the value?

3. Do you take any pain medications? These include Percocet, Percodan, Demerol, Tylox, Tylenol \#3, oxycodone, duragesic (Fentynal) patch, Methadone, and others. No Yes

If yes, which one(s)?

How often do you take this?

When did you last take this type of medicine?

4. Do you take any medications to speed up your GI tract - stomach or colon? These include medications such as Reglan, Zelnorm, Domperidone, and erythromycin. No Yes

If yes, which one(s)?

When did you last take this type of medicine?

5. List any other medications you currently take:

6. Have you had surgery on your GI tract - the esophagus, stomach, or colon? No Yes

If yes, please describe.

\section{ACKNOWLEDGMENT}

This article is reprinted from the American Journal of Gastroenterology (69) with the permission of WileyBlackwell Publishing Ltd.

\section{REFERENCES}

1. Maurer AH, Parkman HP. Update on gastrointestinal scintigraphy. Semin Nucl Med 2006;36:110-8.

2. Griffith GA, Owen GM, Kirkman S, et al. Measurement of the rate of gastric emptying using chromium-51. Lancet 1966;1:1244-5.

3. Camilleri M, Hasler W, Parkman HP, et al. Measurement of gastroduodenal motility in the GI laboratory. Gastroenterology 1998;115:747-62.

4. Parkman HP, Hasler WL, Fisher RS. American Gastroenterological Association technical review on the diagnosis and treatment of gastroparesis. Gastroenterology 2004;127:1592-622.

5. Donohoe KJ, Maurer AH, Ziessman HA, et al. Procedure guideline for gastric emptying and motility - Society of Nuclear Medicine. J Nucl Med 1999;40: 1236-9.

6. Donohoe KJ, Maurer AH, Ziessman HA, et al. Society of Nuclear Medicine. Web site: http://interactive.snm.org/docs/pg_ch08_0403.pdf. Accessed 10 Nov 2007.

7. Lin HC, Prather C, Fisher RS, et al. The AMS Task Force Committee on Gastrointestinal Transit. Measurement of Gastrointestinal Transit. Dig Dis Sci 2005;50:989-1004.

8. Soykan I, Sivri B, Sarosiek I, et al. Demography, clinical characteristics, psychological profiles, treatment, and long-term follow-up of patients with gastroparesis. Dig Dis Sci 1998;43:2398-404.

9. Tack J, Talley NJ, Camilleri M, et al. Functional gastroduodenal disorders. Gastroenterology 2006;130:1466-79.

10. Stanghellini V, Tosetti C, Paternico A, et al. Risk indicators of delayed gastric emptying of solids in patients with functional dyspepsia. Gastroenterology 1996; 110:1036-42.

11. Sarnelli G, Caenepeel P, Geypens B, et al. Symptoms associated with impaired gastric emptying of solids and liquids in functional dyspepsia. Am J Gastroenterol 2003;98:783-8.

12. Jones KL, Wishart JM, Russo A, et al. Predictors of delayed gastric emptying in diabetes. Diabetes Care 2001;24:1264-9. 
13. Samsom M, Vermeijden JR, Smout AJ, et al. Prevalence of delayed gastric emptying in diabetic patients and relationship to dyspeptic symptoms: A prospective study in unselected diabetic patients. Diabetes Care 2003;26:3116-22.

14. Horowitz M, Maddox AF, Wishart JM, et al. Relationships between oesophageal transit and solid and liquid gastric emptying in diabetes mellitus. Eur J Nucl Med 1991;18:229-34.

15. Snape WJ, Battle WM, Schwartz SS, et al. Metoclopramide to treat gastroparesis due to diabetes mellitus. Ann Intern Med 1982;96:444-6.

16. Patterson D, Abell T, Rothstein R, et al. A double-blind multicenter comparison of domperidone and metoclopramide in the treatment of diabetic patients with symptoms of gastroparesis. Am J Gastroenterol 1999;94:1230-4.

17. Revicki DA, Rentz AM, Dubois D, et al. Development and validation of a patient-assessed gastroparesis symptoms severity measure: The Gastroparesis Cardinal Symptom Index. Aliment Pharmacol Ther 2003;18:141-50.

18. Revicki DA, Rentz AM, Dubois D, et al. Gastroparesis Cardinal Symptom Index (GCSI): Development and validation of a patient-reported assessment of severity of gastroparesis symptoms. Qual Life Res 2004;13:833-44.

19. Revicki DA, Rentz AM, Tack J, et al. Responsiveness and interpretation of a symptom severity index specific to upper gastrointestinal disorders. Clin Gastroenterol Hepatol 2004;2:769-77.

20. Hasler WH. Dumping syndrome. Curr Treat Options Gastroenterol 2002;5:139-45.

21. Eagon JC, Miedema BW, Kelly KA. Postgastrectomy syndromes. Surgical Clin North Am 1992;72:445-65.

22. Lin HC, Van Citters GW, Zhao XT, et al. Fat intolerance depends on rapid gastric emptying. Dig Dis Sci 1999;44:330-5.

23. Delgado-Aros S, Camilleri M, Cremonini F, et al. Contributions of gastric volumes and gastric emptying to meal size and postmeal symptoms in functional dyspepsia. Gastroenterology 2004;127:1685-94.

24. Schwartz JG, Green GM, Guan D, et al. Rapid gastric emptying of a solid pancake meal in type II diabetic patients. Diabetes Care 1996;19:468-71.

25. Namin F, Jitan P, Joker I, et al. Clinical hallmarks of cyclic vomiting syndrome (CVS) in adults and role of long-term tricyclic therapy. Gastroenterology 2006;130(Suppl 2):A601.

26. Fajardo NR, Locke GR, Talley NJ. Cyclic vomiting syndrome is associated with rapid early gastric emptying. Am J Gastroenterol 2005;100:143.

27. Lawal A, Barboi A, Krasnow A, et al. Rapid gastric emtpying is more common than gastroparesis in patients with autonomic dysfunction. Am J Gastroenterol 2007;102:618-23.

28. Christian PE, Datz FL, Sorenson JA, et al. Technical factors in gastric emptying studies. J Nucl Med 1983;24:264-8.

29. Nielsen OH, Hvid-Jacobsen K, Lund P, et al. Gastric emptying and subjective symptoms of nausea: Lack of effects of a 5-hydroxytryptamine-3 antagonist ondansetron on gastric emptying in patients with gastric stasis syndrome. Digestion 1990;46:89-96.

30. Nowak A, Jondeerko K, Kaezor R, et al. Cigarette smoking delays gastric emptying of a radiolabelled solid food in healthy smokers. Scand J Gastroenterol 1987;22:54-8.

31. Miller G, Palmer KR, Smith B, et al. Smoking delays gastric emptying of solids. Gut 1989;30:50-3.

32. Wong PWK, Kadakia SC, McBiles M. Acute effect of nicotine patch on gastric emptying of liquid and solid contents in healthy subjects. Dig Dis Sci 1999;44: 2165-71.

33. Sanaka, M, Anjiki H, Tsutsumi H, et al. Effect of cigarette smoking on gastric emptying of solids in Japanese smokers: A crossover study using the 13Coctanoic acid breath test. J Gastroenterol 2005;40:578-82.

34. Schvarcz E, Palmer M, Aman J, et al. Physiological hyperglycemia slows gastric emptying in normal subjects and patients with insulin-dependent diabetes mellitus. Gastroenterology 1997;113:60-6.

35. Horowitz M, O'Donovan D, Jones KL, et al. Gastric emptying in diabetes: Clinical significance and treatment. Diabet Med 2002;19:177-94.

36. Fraser R, Horowitz M, Maddox A, et al. Hyperglycaemia slows gastric emptying in type 1 diabetes mellitus. Diabetologia 1990;30:675-80.

37. Datz FL, Christian PE, Moore J. Gender-related differences in gastric emptying. J Nucl Med 1987;28:1204-7.

38. Gill RC, Murphy PD, Hooper HR, et al. Effect of the menstrual cycle on gastric emptying. Digestion 1987;36:168-74.

39. Tougas G, Eaker EY, Abell TL, et al. Assessment of gastric emptying using a low fat meal: Establishment of international control values. Am J Gastroenterol 2000; 95:1456-62.

40. Gonenne J, Esfandyari T, Camilleri M, et al. Effect of female sex hormone supplementation and withdrawal on gastrointestinal and colonic transit in postmenopausal women. Neurogastroenterol Motil 2006;18:911-8.

41. Chaudhuri TK, Fink S. Gastric emptying in human disease states. Am J Gastroenterol 1991;86:533-8.
42. House A, Champion MC, Chamberlain M. National survey of radionuclide gastric emptying studies. Can J Gastroenterol 1997;11:317-21.

43. Guo JP, Maurer AH, Urbain JL, et al. Extending gastric emptying scintigraphy from two to four hours detects more patients with gastroparesis. Dig Dis Sci 2001;46:24-9.

44. Ziessman HA, Bonta DV, Goetze S, et al. Experience with a new simplified and standardized four-hour gastric emptying protocol. J Nucl Med 2007;48:568-72.

45. Cremonini F, Mullan BP, Camilleri M, et al. Performance characteristics of scintigraphic transit measurements for studies of experimental therapies. Aliment Pharmacol Ther 2002;16:1781-90.

46. Vijayamkumar V, Briscoe EG, Boysen TL, et al. Assessment of the practical role of a low-fat meal solid gastric emptying study. J Nucl Med Technol 2006;34: $82-5$.

47. Camilleri M, Zinsmeister AR, Greydanus MP, et al. Towards a less costly but accurate test of gastric emptying and small bowel transit. Dig Dis Sci 1991;36: 609-15.

48. Thomforde GM, Camilleri M, Phillips SF, et al. Evaluation of an inexpensive screening scintigraphic test of gastric emptying. J Nucl Med 1995;36:93-6.

49. Goetze S, Ravich WJ, Ziessman HA. Increased detection of abnormal gastric emptying at 4 hours compared to two hours using a standardized protocol. J Nucl Med 2005; 46:320.

50. Abell TL, Starkebaum W, Abidi N, et al. How common is rapid gastric emptying in gastroparesis. Gastroenterology 2006;130 (Suppl 2):A244-5.

51. Lin Z, Sarosiak I, McCallum RW. Optimal detection of gastroparesis. Am J Gastroenterol 2006;101:S135.

52. Arts J, Caenepeel P, Verbeke K, et al. Influence of erythromycin on gastric emptying and meal-related symptoms in functional dyspepsia with delayed gastric emptying. Gut 2005;54:455-60.

53. Camilleri M. Clinical practice. Diabetic gastroparesis. N Engl J Med 2007;356: 820-951.

54. Fich A, Neri M, Camilleri M, et al. Stasis syndromes following gastric surgery: clinical and motility features of 60 symptomatic patients. J Clin Gastroenterol 1990;12:505-12.

55. Miedema BW, Kelly KA, Camilleri M, et al. Human gastric and jejunal transit and motility after Roux gastrojejunostomy. Gastroenterology 1992;103:1133-43.

56. Troncon LE, Bennett RJ, Ahluwalia NK, et al. Abnormal intragastric distribution of food during gastric emptying in functional dyspepsia patients. Gut 1994;35:327-32.

57. Gonlachanvit S, Maurer AH, Fisher RS, et al. Regional gastric emptying abnormalities in functional dyspepsia and gastroesophageal reflux disease. Neurogastroenterol Motil 2006;18:894-904.

58. Karamanolis G, Caenepeel P, Arts J, et al. Determinants of symptom pattern in idiopathic severely delayed gastric emptying: Gastric emptying rate or proximal stomach dysfunction? Gut 2007;56:29-36.

59. Piessevaux H, Tack J, Wairand S, et al. Intragastric distribution of a standardized meal in health and functional dyspepsia: Correlation with specific symptoms. Neurogastroenterol Motil 2003;15:447-55.

60. Ziessman HA, Fahey FH, Collen MJ. Biphasic solid and liquid gastric emptying in normal controls and diabetics using continuous acquisition in LAO view. Dig Dis Sci 1992;37:744-50.

61. Camilleri M, Brown ML, Malagelada JR. Relationship between impaired gastric emptying and abnormal gastrointestinal motility. Gastroenterology 1986;91:94-9.

62. Mackie AD, Ferrington C, Cowan S, et al. The effects of renzapride, a novel prokinetic agent, in diabetic gastroparesis. Aliment Pharmacol Ther 1991;5:135-42.

63. Elashoff JD, Reedy TJ, Meyer JH. Methods for analyzing gastric emptying. Am J Physiol 1983;244:G701-2.

64. Siegel JA, Urbain JL, Adler LP, et al. Biphasic nature of gastric emptying. Gut 1988;29:85-9.

65. Nusynowitz ML, Benedetto AR. The lag phase of gastric emptying: Clinical, mathematical and in vitro studies. J Nucl Med 1994;35:1023-7.

66. Camilleri M, Malagelada JR, Brown ML, et al. Relation between antral motility and gastric emptying of solids and liquids in humans. Am $\mathrm{J}$ Physiol 1985;249:G580-5.

67. Salaun PY, Querellouu S, Nguyen JM, et al. Comparison of gastric emptying scintigraphy based on the geometric mean of the gastric proportion of the abdominal radioactivity or on the geometric mean of the intragastric radioactivity. Nucl Med Commun 2006;27:431-7.

68. Lien H, Chang C, Chen G, et al. Gastric emptying rate assessment based on the proportion of intra-abdominal radioactivity in the stomach. J Nucl Med 1999; 40:1106-10.

69. Abell TL, Camilleri M, Donohoe K, et al. Consensus recommendations for gastric emptying scintigraphy: a joint report of the American Neurogastroenterology and Motility Society and the Society of Nuclear Medicine. Am J Gastroenterol 2007; 102:1-11. 


\section{CONFLICT OF INTEREST}

Guarantor of the article: Henry P. Parkman, M.D.

Specific author contributions: Thomas L. Abell, Richard W. McCallum, Lawrence A. Szarka, William J. Snape Jr, and Harvey A. Ziessman: presented information at meeting leading to formulation of the manuscript and reviewed the manuscript several times with useful suggestions that were incorporated; Michael Camilleri: contributed to the discussions of consensus opinions and revised manuscript several times; Kevin Donohoe: presented information at the two meetings leading to formulation of the manuscript and reviewed the manuscript several times with suggestions that were incorporated; William L. Hasler, Thomas Nowak, Martin L. Nusynowitz, and Paul Shreve: presented information at the meeting leading to formulation of the manuscript and reviewed the manuscript with suggestions; Henry C. Lin: presented information at the meeting leading to formulation of the manuscript; and Alan H. Maurer and Henry P. Parkman: coorganizers of the project. They helped develop the initial draft of the manuscript and revised the manuscript multiple times.

Financial support: Financial support was received to help support the two meetings of the authors that led to the development of the manuscript. The following organizations provided support for the meetings, but did not provide input into the development of the manuscript: Gastroparesis \& Dysmotilities Association (GPDA); Novartis Pharmaceuticals, Inc; TAP Pharmaceuticals, Inc; and General Electric, Inc.

Potential competing interests: None. 